\title{
Uso integral de simulación, diseño de experimentos y KANBAN para evaluar y mejorar el rendimiento de una línea de producción ${ }^{1}$
}

\section{Integral use of simulation, design of experiments and KANBAN to evaluate and improve the performance of a production line}

\author{
S. A. Fernández, A. L. Pérez y P. D. Medina \\ Recibido: noviembre 14 de 2018 - Aceptado: septiembre 30 de 2019
}

\begin{abstract}
Resumen-Este artículo es el producto de una investigación desarrollada en una línea de producción, donde se quería observar como el Diseño de Experimentos (DOE) y el KANBAN interactúan integralmente con la Simulación Discreta en pro de mejorar el rendimiento del sistema productivo. Lo anterior, se logró mostrando la forma en que el DOE sirve para identificar los factores relevantes en el proceso productivo, para luego, ser incorporados como parámetros de entrada en la simulación e igualmente, a través de la implementación de la herramienta KANBAN tipo "Trigger" se evidenció las mejoras de desempeño que se presentan en dicha línea de producción. Con base a lo anterior, se realizaron dos simulaciones: Una sin el uso de la herramienta Lean y otra con su implementación. De esta manera, se pudo observar el mejoramiento en el desempeño de la línea de producción al incorporar el KANBAN. Por ejemplo, el proceso de "Planchado" que tenía un porcentaje de utilización del $36 \%$, aumentó a 60,39\% con el uso del Kanban, lo mismo le ocurrió al proceso de "Tejido" que aumentó su utilización hasta un $61,77 \%$. De esta manera, se deja ilustrada una forma de cómo mejorar el rendimiento de una línea de producción soportado en la combinación de estas tres herramientas.
\end{abstract}

${ }^{1}$ Producto derivado del proyecto de investigación "Plan de mejoramiento de la línea de producción de la empresa tejidos de Risaralda utilizando Diseño de Experimentos y Simulación Discreta que permitan el uso óptimo de los recursos y la minimización de los costos", apoyado por la Universidad Tecnológica de Pereira a través de la Maestría en Investigación Operativa y Estadística.

S. A. Fernández, Politécnico Colombiano Jaime Isaza Cadavid, Medellín, Colombia, email: safernandez@elpoli.edu.co.

A. L. Pérez, Universidad Tecnológica de Pereira, Pereira, Colombia, email: lliceth@utp.edu.co

P. D. Medina, Universidad Tecnológica de Pereira, Pereira, Colombia, email: pemedin@utp.edu.co.

Como citar este artículo: Fernández, S. A., Pérez, A. L., y Medina, P. D. Uso integral de simulación, diseño de experimentos y KANBAN para evaluar y mejorar el rendimiento de una línea de producción, Entre Ciencia e Ingeniería, vol. 13, no. 26, pp. 9-16, julio-diciembre 2019. DOI: https://doi.org/10.31908/19098367.1147

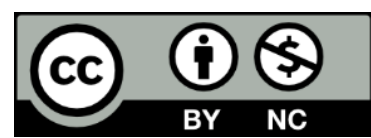

Attribution-NonCommercial 4.0 Intenational (CC By-NC 4.0)
Palabras clave- Simulación Discreta, Diseño de Experimentos, KANBAN tipo TRIGGER.

Abstract - This article is the product of research developed in a production line, where we wanted to observe how the Design of Experiments (DOE) and the Kanban interact fully with the Discrete Simulation in order to improve the performance of the production system. The above was achieved by showing how the DOE serves to identify the relevant factors in the production process, to then be incorporated as input parameters in the simulation, and also, through the implementation of the Kanban tool type "Trigger" the performance improvements presented in such production line were evidenced. Based on the above, two simulations were made: One without the use of the Lean tool and another with its implementation. In this way, the improvement in the performance of the production line could be observed when incorporating the Kanban. For example, the "Ironing" process, which had a utilization rate of $36 \%$, increased to $60.39 \%$ with the use of Kanban, the same thing happened to the "Weaving" process, which increased its use to $61.77 \%$. In this way, a way to improve the performance of a production line supported in the combination of these three tools is illustrated.

Descriptors - Discrete Simulation, Design of Experiments, KANBAN type TRIGGER.

\section{INTRODUCCIÓN}

$\mathrm{S}$ E sabe que hay empresas que deben fabricar en cantidades variables y con una demanda dinámica una amplia diversidad de productos para satisfacer mercados más exigentes con relación a altos estándares de calidad [1], sin que esto suponga un incremento considerable en el precio final. Debido a ello, se debe contemplar estrategias de fabricación que involucren como uno de sus objetivos fundamentales aumentar la flexibilidad en la producción. Adicionalmente, antes de implementar dichas estrategias es recomendable hacer pruebas de su pertinencia para no incurrir en costos innecesarios por una decisión mal tomada.

Teniendo en cuenta que los modelos de simulación discreta son una herramienta importante que permiten explicar los procesos que se presentan en cualquier empresa de manufactura que involucran procesos de tiempos, de producción, inventarios, y otros [2], [3]; y que esta simulación 
discreta ha ido ganando importancia a nivel empresarial, ya que permite la representación, análisis y experimentación del sistema producción considerando condiciones de incertidumbre [4], se utilizó este tipo de simulación en la investigación desarrollada para tener una aproximación real a una de las líneas de producción de una empresa de tipo textil dado su importancia en el sector industrial colombiano [5].

Con esta simulación se buscó establecer cuáles eran los factores que retrasan la producción y cuáles eran los puntos críticos de dicho proceso [5], para poder generar un plan de mejoramiento que pueda flexibilizar el flujo productivo y aumentar la capacidad de respuesta para la demanda variable.

Además de hacer uso de la simulación discreta, este estudio también se apoyó en la "manufactura esbelta", dado que, además de contar con una cantidad relevante de "Herramientas Lean", su uso genera un impacto relevante en la productividad de la empresa [6], puesto que ayuda a disminuir todas las mudas que pueda presentar el proceso de fabricación.

En la lista de "Herramientas Lean" se encuentra el KANBAN que puede utilizarse como un sistema de información que controla la cantidad y tiempo de producción de los elementos que serán fabricados tanto en la planta como en el exterior en pro de optimizar los procesos [7], y obtener un sistema justo a tiempo [8]. Por lo tanto, este estudio también se soportó en dicha herramienta aplicando kanban tipo trigger [9], para que el modelo propuesto propenda por la disminución de los tiempos de procesamiento [10], en pro de la reducción de costos y flexibilización del sistema productivo soportado en el jalonamiento de los materiales desde la orden del cliente.

Igualmente, esta investigación utilizó herramientas robustas de estadística como lo es Diseño de Experimentos (DOE), dado que en estudios [11], se ha presentado la importancia de incorporar DOE [12], a la simulación para analizar la interacción de ciertas variables de estudio y poder obtener un mejor modelo de simulación con un menor esfuerzo computacional [13], donde integran la simulación, el DOE y las Metaheurísticas para optimizar la productividad en un sistema de manufactura [14], se incorporó DOE en los procesos críticos de la línea de producción objeto de estudio para determinar la significancia de los operarios en los tiempos de operación, para así, incluir de dichos tiempos de operación, los valores más idóneos en el modelo de simulación discreta, logrando de esta manera, una simulación con un tiempo de fabricación acorde al mejor rendimiento de los operarios.

Este artículo se organiza de la siguiente forma: en la metodología se mencionan las tres fases de la investigación, el flujograma del proceso, el diseño experimental con los factores y niveles utilizados, el modelo de simulación discreta y la metodología Kanban tipo Trigger Aplicada; en los resultados se muestran las tablas ANOVA, las gráficas utilizando el software minitab, los cuadros con las pruebas de Tukey y el cuadro comparativo entre los dos modelos aplicados sin Kanban y con Trigger. Por último, se presentan las conclusiones en donde se refleja la importancia de incorporar las herramientas Lean a la hora de proponer mejoras en un sistema de producción.

\section{METODOLOGÍA}

Esta investigación se desarrolló en cuatro fases, luego de las cuales, se logró el mejoramiento del rendimiento de la línea de producción de la empresa caso de estudio, soportado en los resultados obtenidos por la implementación de Diseño de Experimentos, Simulación Discreta y la técnica Kanban tipo Trigger.

Fase I, con el objetivo de realizar una investigación con datos reales y evidenciar la utilidad de la integración de las tres técnicas (Simulación, DOE y Kanban), se realizaron visitas a una empresa del sector textil, en donde, se obtuvo la descripción del flujo de las actividades que se realizan en su línea de producción y la recolección de la información necesaria para los respectivos análisis, como lo son los tiempos en las diferentes zonas de procesamiento, el registro de los pedidos realizados por los clientes, tiempos de transporte, entre otros. La tabla I presenta un resumen del Flujograma de la línea en la que se hizo la investigación.

Fase II, dado que para la simulación de la línea de producción se desea contar con tiempos de operación idóneos que ayuden a obtener resultados de buena calidad respecto al tiempo total de fabricación y unidades entregadas, se aplicó un modelo de Diseño de Experimentos (DOE) en los tres procesos más relevantes del sistema, en donde, cada uno cuenta con dos operarios para que realicen el proceso respectivo. De esta manera, se planteó un diseño factorial 23 en busca de identificar sí los operarios inciden significativamente en el tiempo de procesamiento de sus operaciones asignadas, en pro de determinar el mejor rendimiento en cada proceso y con esta información realizar un comparativo con los resultados del modelo de Simulación Discreta para generar un plan de acción de buena calidad, tal como se mencionó anteriormente.

La tabla II presenta los factores con sus respectivos niveles, en donde, cada factor hace referencia a uno de los tres procesos relevantes del sistema y cada nivel indica el operario que puede ser asignado al proceso respectivo. Por ejemplo, en el proceso de "Preparación (A)" cuenta con dos niveles que son los dos operarios que están encargados para esta labor (OP_1 y OP_2), lo mismo ocurre para los otros dos procesos.

La variable de respuesta es el "Tiempo de procesamiento" tomado en minutos. En la tabla III se observa el resumen de la configuración para el DOE mencionado anteriormente con 8 tratamientos y 3 réplicas.

Fase III, se elaboró el modelo de Simulación Discreta de la situación actual de la empresa, utilizando la información obtenida en la Fase I, evaluando la fiabilidad y validez de la información suministrada por los directivos de la empresa y los investigadores en cuestión. Cabe resaltar que la simulación se corrió por 192 horas que equivalen a un mes laboral en la empresa, además, se tuvo 50 réplicas para trabajar con los datos promedios de la estadística generada logrando así, una mayor confiabilidad en los datos estimados y bajo un sistema "No Terminante", puesto que la producción se continuaba día tras día en los que se había parado el día anterior. 
TABLA I.

FLUJOGRAMA DEL PROCESO PRODUCTIVO.

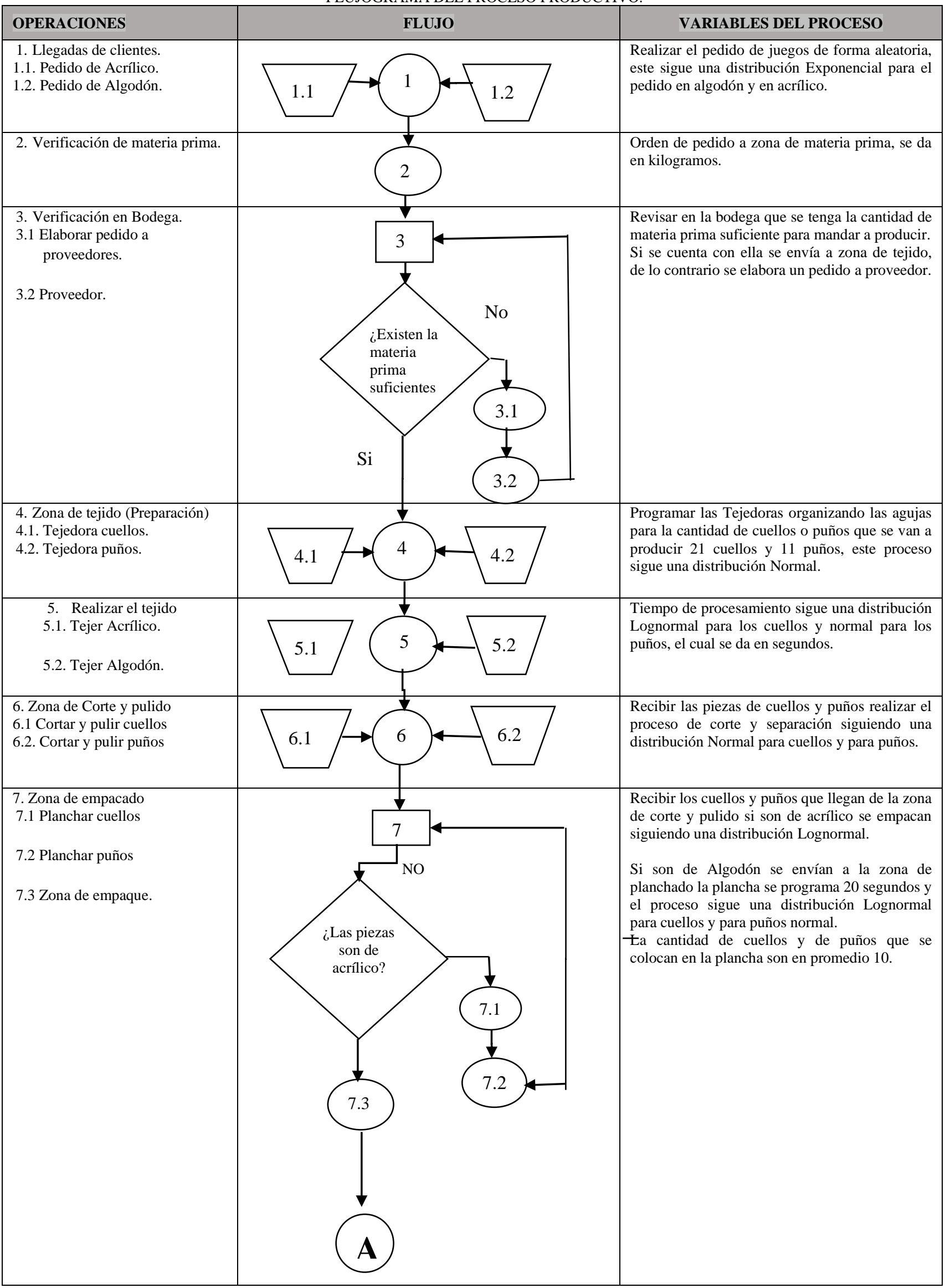




\begin{tabular}{|l|l|l|}
\hline OPERACIONES & VARIABLES DEL PROCESO \\
\hline $\begin{array}{l}\text { 8. Inspección final } \\
\text { 8.1 Evaluar pedido } \\
\text { suficiente } \\
\text { 8.2 Completar el pedido } \\
\text { 8.3 Llevar a zona de } \\
\text { producto terminado } \\
\text { 8.4 Entrega. }\end{array}$ & $\begin{array}{c}\text { Revisar que se tenga el pedido completo, se } \\
\text { organizan paquetes de 100 unidades de puños y } \\
\text { cuellos, si no se tiene se espera que se complete } \\
\text { y se envía a la zona de producto terminado. }\end{array}$ \\
\hline
\end{tabular}

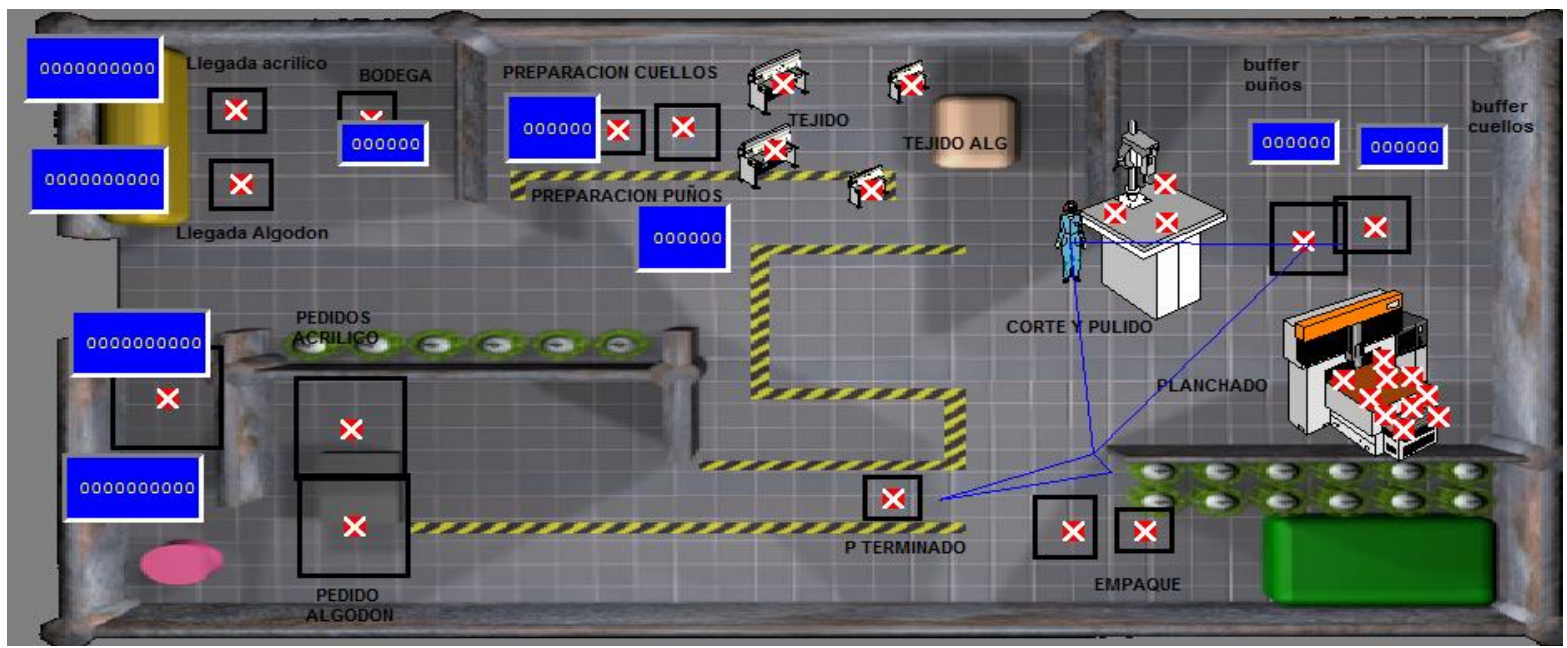

Fig. 1. Layout del proceso productivo objeto de estudio. Software PROMODEL.

TABLA II.

FACTORES Y NIVELES DEL DISEÑO DE EXPERIMENTOS.

\begin{tabular}{|c|c|}
\hline FACTORES & NIVELES \\
\hline \multirow{2}{*}{ Preparación (A) } & OP_1 \\
\cline { 2 - 2 } & OP_2 \\
\hline \multirow{2}{*}{ Tejedora (B) } & Tej_1 \\
\cline { 2 - 2 } & Tej_2 \\
\hline \multirow{2}{*}{ Cortadora (C) } & COP_1 \\
\cline { 2 - 2 } & COP_2 \\
\hline
\end{tabular}

La Fig. 1 presenta la forma como se diseñó el "Layout" del proceso productivo, con base a la información citada en la tabla I. Donde se representa el proceso productivo de la empresa.

Fase IV, se aplicó la técnica Kanban tipo Trigger buscando obtener mejoras en la línea de producción de la empresa a partir de los resultados obtenidos en la Fase II y III. Para ello, se consideró una propuesta de mejora que permite incrementar su desempeño actual donde la técnica "dispara" la orden de producción cuando llega el pedido.

Para realizar el modelo de Simulación Discreta aplicando esta metodología Kanban se utilizó el "Trigger" en el pedido de algodón donde se presenta mayor retraso y no se alcanzan a unir la misma cantidad de cuellos y puños para formar el juego.

\section{RESULTADOS}

Al aplicar el DOE para el tiempo de procesamiento propuesto en la fase II de esta investigación, se observó mediante el resumen del ANOVA respectivo que las interacciones del modelo factorial implementado no eran significativas, por lo que, se volvió a correr el modelo sin dichas interacciones.

En la ANOVA presentada en la tabla IV, se observa que en los procesos de "Preparación" y "Cortadora" es donde los operarios presentan diferencias significativas en sus tiempos de procesamiento. Además, se concluye que en la tejedora el tiempo de operación es estadísticamente igual para los dos operarios que hacen esta labor.

Con base en los resultados del ANOVA y el comportamiento de los efectos principales presentados en la gráfica 1 , se evidencia que el operario 2 de "Preparación" y el cortador 2 de "Cortadora" son los que presentan un mejor tiempo de procesamiento.

Se verificaron los supuestos del modelo de análisis de varianza donde se encontró que las varianzas de los tratamientos son iguales utilizó la prueba de Bartlett y de 
Levene, dado que el valor-p en ambas pruebas es mayor que el nivel de significancia (0.05); los datos también se ajustaron a una distribución normal se utilizó la prueba de AndersonDarling y dado que el valor-p es mayor que el nivel de significancia $(0.16>0.05)$.

Finalmente, para terminar de comprobar la veracidad de la información presentada en la Fig. 2, se utilizó la prueba de Tukey para los dos factores en cuestión con el objetivo de comprobar si los tiempos promedio de operación realizados por cada uno de los operarios asignados a las tareas en cuestión eran estadísticamente iguales.

TABLA III.

CONFIGURACIÓN DE DOE FACTORIAL $2^{3 *}$

\begin{tabular}{|c|c|c|c|}
\hline PREPARACION & TEJEDORA & CORTADORA & \begin{tabular}{|c}
$\begin{array}{c}\text { Tiempo de procesamiento } \\
\text { (Minutos) }\end{array}$ \\
\end{tabular} \\
\hline OP_1 & Tej_1 & COP_1 & 8.828 \\
\hline OP_1 & Tej_2 & COP_1 & 8.418 \\
\hline OP_1 & Tej_1 & COP_2 & 7.828 \\
\hline OP_1 & Tej_2 & COP_2 & 7.418 \\
\hline $\mathrm{OP} \_2$ & Tej_1 & COP_1 & 9.128 \\
\hline OP_2 & Tej_2 & COP_1 & 8.718 \\
\hline $\mathrm{OP} \_2$ & Tej_1 & COP_2 & 8.128 \\
\hline OP_2 & Tej_2 & COP_2 & 7.718 \\
\hline OP_1 & Tej_1 & COP_1 & 8.885 \\
\hline OP_1 & Tej_2 & COP_1 & 8.905 \\
\hline OP_1 & Tej_1 & COP_2 & 8.325 \\
\hline OP_1 & Tej_2 & COP_2 & 8.345 \\
\hline $\mathrm{OP} \_2$ & Tej_1 & COP_1 & 7.705 \\
\hline OP_2 & Tej_2 & COP_1 & 7.725 \\
\hline $\mathrm{OP} \_2$ & Tej_1 & COP_2 & 7.145 \\
\hline $\mathrm{OP} \_2$ & Tej_2 & COP_2 & 7.165 \\
\hline OP_1 & Tej_1 & COP_1 & 9.318 \\
\hline OP_1 & Tej_2 & COP_1 & 9.324 \\
\hline OP_1 & Tej_1 & COP_2 & 8.208 \\
\hline OP_1 & Tej_2 & COP_2 & 7.384 \\
\hline OP_2 & Tej_1 & COP_1 & 8.488 \\
\hline OP_2 & Tej_2 & COP_1 & 8.494 \\
\hline $\mathrm{OP} \_2$ & Tej_1 & COP_2 & 7.378 \\
\hline OP_2 & Tej_2 & COP_2 & 7.384 \\
\hline
\end{tabular}

*Tomado del DOE planteado por el software Minitab 17.

Con los datos de la tabla V, se puede corroborar la diferencia significativa entre los tiempos de los dos operarios de cada actividad y además, se verifica que el OP_2 en la "Preparación" y el COP_2 en la "Cortadora" presentan el menor tiempo de procesamiento, dado el comportamiento de la diferencia entre medias mostrado en la segunda columna.

La información obtenida con este DOE es muy importante porque sirve para ser tomada en cuenta en el modelo de simulación como parámetro de entrada en pro de generar una solución de buena calidad soportada en los mejores tiempos de procesamiento, que, para este caso, será tomar los tiempos del operario 2 (OP_2) de Preparación y operario 2 (COP_2) de Cortadora para dicha simulación. Además, también les sirve a los directivos de la empresa para iniciar un proceso de capacitación que busque como resultado que los demás operarios lleguen a tener el mismo rendimiento de los dos operarios en cuestión.
La tabla VI presenta los tiempos de los operarios elegidos con base al DOE para cada uno de los procesos en la línea de fabricación objeto de estudio.

TABLA IV

Resultados ANOVA sin efectos de Interacción.

\begin{tabular}{|lcl}
\hline Factor & Tipo & Niveles Valores \\
Preparación fijo & 2 & Operario_1; Operario_2 \\
Tejedora fija & 2 & Tejedora_1; Tejedora_2 \\
Cortadora fijo & 2 & Cortar_1; Cortar_2
\end{tabular}

Análisis de varianza para Tiempo_procesamiento, utilizando SC ajustada para pruebas

Fuente GL SC Sec. SC Ajust. CM Ajust. F P $\begin{array}{lllllll}\text { Preparación } & 1 & 2,2467 & 2,2467 & 2,2467 & 11,31 & 0,003\end{array}$ $\begin{array}{lllllll}\text { Tejedora } & 1 & 0,0323 & 0,0323 & 0,0323 & 0,16 & 0,691\end{array}$ $\begin{array}{lllllll}\text { Cortadora } & 1 & 4,8988 & 4,8988 & 4,8988 & 24,67 & 0,000\end{array}$ $\begin{array}{lllll}\text { Error } & 19 & 3,7733 & 3,7733 & 0,1986\end{array}$ Total $23 \quad 11,1751$

En la Fase III, elaboración del modelo de Simulación, se realizó la programación del modelo de Simulación involucrando todos los procesos y actividades que se llevan a cabo en la línea de producción de la empresa objeto de estudio. Durante la elaboración de dicho modelo se realizaron varios ajustes a partir de la creación de variables, locaciones o entidades para la correcta programación, tal como se especifica a continuación:

- La elaboración de los juegos en acrílico y algodón tiene dos políticas, primero se mandan a fabricar las piezas de los cuellos y luego la de los puños, además un $70 \%$ del producto fabricado es de algodón y el 30\% restante de acrílico. (Esta información fue obtenida de los históricos de la empresa de las ventas de los últimos años).

- Las piezas de cuello y puños elaboradas en algodón se deben planchar y las realizadas en acrílico no deben pasar por esta estación, van directamente a la zona de empaque.

- Para mandar a empacar se deben completar 100 piezas de cuellos o de puños y luego son enviadas a la estación de empaque.

- El modelo de Simulación Discreta se implementó en un tiempo real para establecer las variables que presentan mayor retraso en los tiempos de procesamiento.

- La opción dada para el tiempo de corrida fue de 192 horas que equivale a un mes laboral en la empresa. Cabe resaltar que el sistema se comporta de una forma no terminante, es decir, cuando se acaba un turno para la producción se detiene el proceso, pero al día siguiente se continúa con el proceso en el que se llevaba el pedido, lo que indica que es un sistema continuo.

Al aplicar el modelo de Simulación Discreta en la línea de producción de la empresa, se evidenciaron algunos problemas con respecto a cuellos de botella, la poca utilidad de algunas locaciones, los retrasos en los tiempos de producción y el tiempo ocioso de los operarios. Lo cual, se reflejaba en la elaboración del producto terminado especialmente en los pedidos de algodón. Con la implementación de la metodología Kanban tipo Trigger se pudo observar que la mayoría de estos 


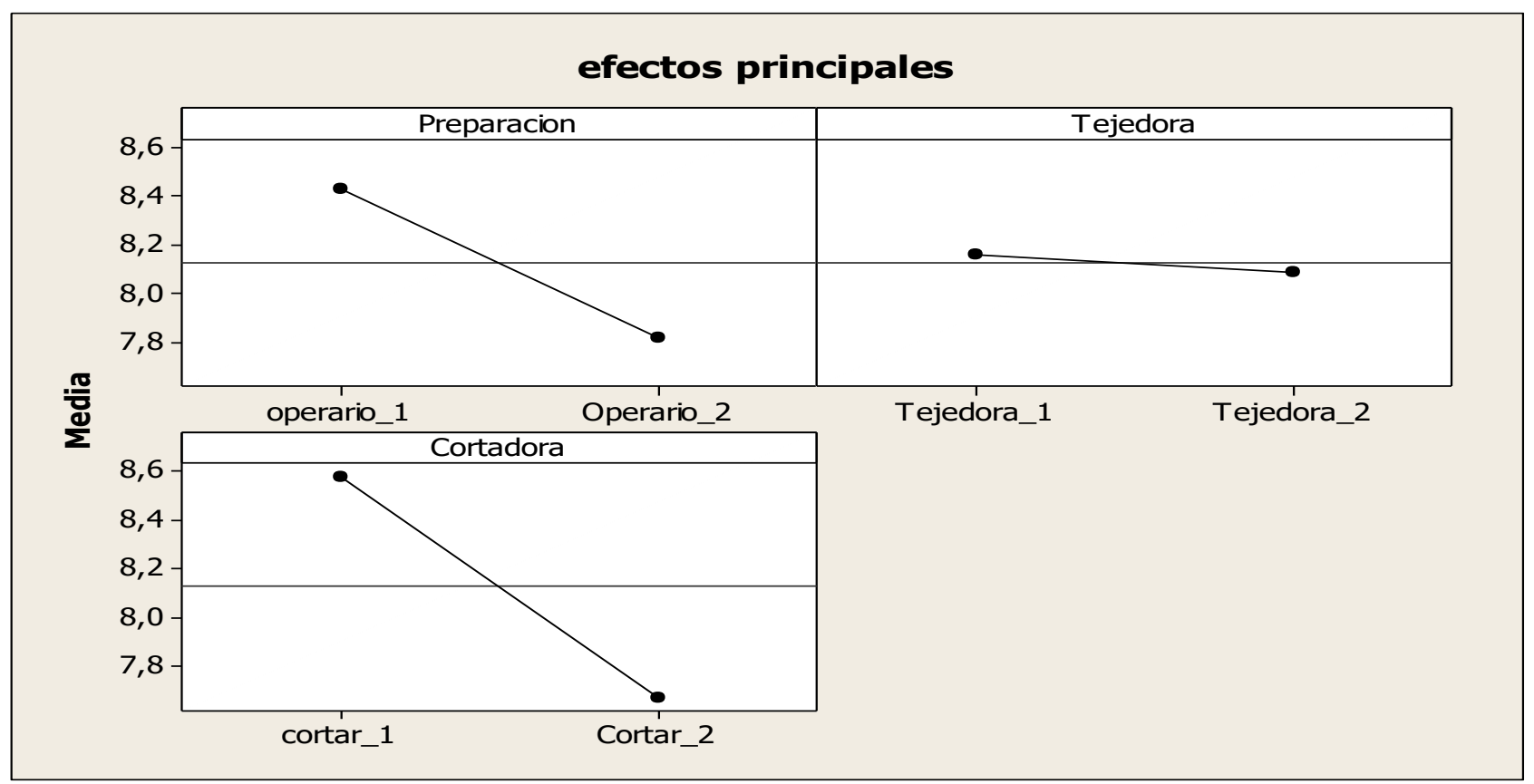

Fig. 2. Comportamiento Efectos Principales. (Resultado en el software Minitab 17).

problemas se redujeron como se muestra en la tabla VII, donde se hace una comparación entre los dos tipos de simulaciones, evidenciando así, las ventajas de haber reaizado este estudio soportado en las tres técnicas tratadas (DOE, Simulación y Kanban tipo Trigger).

\section{CONCLUSIONES}

Los resultados obtenidos en la investigación permiten establecer que al unir varias metodologías como el DOE, la Simulación Discreta y herramientas Lean (en este caso Kanban), se pueden identificar claramente los factores que inciden en los retrasos de los procesos, además de permitir incorporar en una empresa, nuevas y mejores políticas de producción que aumenten el rendimiento de la misma. Esto se pudo comprobar en las simulaciones, donde se obtuvo incrementos en la tasa de producción del $54,7 \%$ siendo un valor relevante para las utilidades de la compañía.

Al apoyar el proceso de Simulación con herramientas estadísticas robustas como el DOE, se logra identificar factores relevantes en el modelo y puntos críticos, donde se debe colocar mayor atención para obtener escenarios que le apuntan a una mayor productividad del caso real a simular. Lo anterior, se validó en el caso simulado, el cual se soportó en los tiempos de proceso relevantes de las dos estaciones que pudo identificar dicha herramienta estadística.

Se pudo comprobar en esta investigación la importancia de incorporar las herramientas Lean a la hora de proponer mejoras en un sistema de producción. Tal es el caso, cuando el modelo de Simulación original presentó un porcentaje de utilidad en el mes del $35.23 \%$, lo que indica que el $64.77 \%$ del tiempo era tiempo muerto, que a su vez se transmite en pérdidas para la empresa reflejados en costos de operaciones entre otros, y al realizar un nuevo modelo de simulación incorporando la herramienta "Kanban Trigger", se aumentó el porcentaje de utilización de los operarios al 77,52\% disminuyendo dicho tiempo muerto considerablemente, aumentando por ende, la productividad de todo el sistema simulado.

\section{DISCUSIONES}

En esta investigación con la información obtenida se logró establecer políticas de mejoras para la línea de producción de la empresa Tejidos de Risaralda, se citará a manera de recomendación la realización de trabajos futuros, relacionados con la investigación realizada, las sugerencias son:

TABLA V

PRUEBA DE TUKEY PARA "PREPARACIÓN" Y "CORTADORA"

\begin{tabular}{|cc|c|c|c|}
\hline $\begin{array}{c}(\mathrm{I}) \\
\text { PREPARA } \\
\text { CIÓN }\end{array}$ & $\begin{array}{c}\text { PREPARA } \\
\text { CIÓN }\end{array}$ & $\begin{array}{c}\text { Diferencia entre } \\
\text { medias (I-J) }\end{array}$ & $\begin{array}{c}\text { Error } \\
\text { típ. }\end{array}$ & $\begin{array}{c}\text { Significa } \\
\text { ción(a) }\end{array}$ \\
\hline OP_1 & OP_2 & 0,501 & 0,179 & $\mathbf{0 , 0 1 1}$ \\
OP_2 & OP_1 & $-0,501$ & 0,179 & $\mathbf{0 , 0 1 1}$ \\
\hline $\begin{array}{c}\text { (I) } \\
\text { CORTA } \\
\text { DORA }\end{array}$ & $\begin{array}{c}\text { CORTA } \\
\text { DORA }\end{array}$ & $\begin{array}{c}\text { Diferencia entre } \\
\text { medias (I-J) }\end{array}$ & $\begin{array}{c}\text { Error } \\
\text { típ. }\end{array}$ & $\begin{array}{c}\text { Significa } \\
\text { ción(a) }\end{array}$ \\
\hline COP_1 & COP_2 &, $959\left(^{*}\right)$ & 0,179 & $\mathbf{0 , 0 0 0}$ \\
COP_2 & COP_1 &,$- 959\left(^{*}\right)$ & 0,179 & $\mathbf{0 , 0 0 0}$ \\
\hline
\end{tabular}


Implementar un modelo combinando metaheurísticas con Kanban personalizados para cada estación de trabajo, aunque para realizar esta aplicación la empresa debe mejorar su registro de información en las diferentes estaciones debido a que fue difícil obtener los datos de pedidos de juegos en acrílico y en algodón de los últimos años porque la empresa no tenía un registro completo y actualizado.

Se recomienda para investigaciones futuras construir KANBANS con diferentes enfoques de transporte, KANBAN CONWIP o de DOBLE TARJETAS que permitan un mejor rendimiento y una disminución de los tiempos de procesamiento.
TABLA VI. TIEMPOS DE PROCESAMIENTO SUGERIDOS

\begin{tabular}{|c|l|l|}
\hline \multicolumn{1}{|c|}{ Locación } & \multicolumn{1}{|c|}{ Entidad } & \multicolumn{1}{c|}{ Distribución } \\
\hline \multirow{2}{*}{ Preparación de Maquina } & Cuellos & N(2, 0.10)MIN \\
\cline { 2 - 3 } & Puños & N(1, 0.10)MIN \\
\hline \multirow{2}{*}{ Corte } & Cuellos & N(4, 0.12)MIN \\
\cline { 2 - 3 } & Puños & N(7, 0.15)MIN \\
\hline
\end{tabular}

TABLA VII COMPARATIVO DE LOS DOS MODELOS DE SIMULACIÓN.

\begin{tabular}{|c|c|c|}
\hline & Modelo Simulado sin Kanban & Modelo Aplicando Kanban Tipo Trigger \\
\hline \multirow{3}{*}{ Locaciones } & $\begin{array}{l}\text { El tiempo promedio en el que están las entidades en cada } \\
\text { locación, muestra que las locaciones Empaque de puños y } \\
\text { cuellos son en las que más tiempo se demoran las } \\
\text { entidades. } 4088,19 \text { y } 3952.60 \text { minutos respectivamente. }\end{array}$ & $\begin{array}{l}\text { Aplicando el Kanban este tiempo disminuyó a } 2855,81 \text { minutos en } \\
\text { empaque puños y } 2815,94 \text { minutos empaque cuellos, lo cual, } \\
\text { representa una reducción del tiempo de espera del } 30,14 \% \text { y } \\
28,9 \% \text { respectivamente. }\end{array}$ \\
\hline & $\begin{array}{l}\text { El porcentaje de utilidad de la locación planchado fue del } \\
39,06 \% \text { y de las maquinas tejedoras de algodón del } \\
43,40 \% \text {, lo que indica que estuvieron una gran parte del } \\
\text { tiempo inutilizadas generando un alto porcentaje de } \\
\text { tiempo ocioso. }\end{array}$ & $\begin{array}{l}\text { Al aplicar la metodología Kanban tipo Trigger facilita que la } \\
\text { materia prima se mueva por las locaciones disminuyendo los } \\
\text { cuellos de botella, lo cual hace que el porcentaje de utilidad de la } \\
\text { locación planchado pase de estar al } 39 \% \text { y aumente hasta un } \\
60,39 \% \text {, lo mismo ocurre con las maquinas tejedoras donde pasa } \\
\text { de un } 43 \% \text { a un } 61,77 \% \text {. }\end{array}$ \\
\hline & $\begin{array}{l}\text { La cantidad de materia prima que llega es de } 12887 \mathrm{~kg} \mathrm{y} \\
\text { con ellos se elaboran únicamente } 10917 \text { juegos quedando } \\
1970 \mathrm{~kg} \text { de materia prima para una nueva producción de } \\
\text { juegos. }\end{array}$ & $\begin{array}{l}\text { Con esta metodología llegaron } 17029 \mathrm{~kg} \text { de materia prima y se } \\
\text { fabricaron } 16532 \text { juegos de algodón. Unicamente se quedaron } \\
\text { para una nueva producción } 497 \mathrm{~kg} \text {, lo que reduce los desperdicios } \\
\text { y la sub-producción. }\end{array}$ \\
\hline Recursos & $\begin{array}{l}\text { El porcentaje de utilización de los operarios es del } \\
35,23 \% \text {, lo que indica que, durante el mes de la } \\
\text { Simulación, un 64,77\% del tiempo de trabajo es } \\
\text { desperdiciado esperando que lleguen las piezas de la } \\
\text { operación anterior en este caso la zona de tejido algodón } \\
\text { para continuar el proceso. }\end{array}$ & $\begin{array}{l}\text { Después de aplicar la metodología Kanban Trigger este porcentaje } \\
\text { de utilización de los operarios aumentó al } 77,52 \% \text {, siendo } \\
\text { únicamente un } 22,48 \% \text { de tiempo ocioso, esto se presenta debido a } \\
\text { que la estación anterior (tejido algodón) aumenta su tiempo de } \\
\text { utilización y las preparaciones de cuellos y puños disminuyeron su } \\
\text { cuello de botella. }\end{array}$ \\
\hline Entidades & $\begin{array}{l}\text { Al finalizar las } 192 \text { horas de Simulación salieron } 10600 \\
\text { cuellos de algodón y } 9600 \text { puños de algodón, quedándose } \\
\text { en el sistema } 317 \text { cuellos y } 6576 \text { puños para completar el } \\
\text { pedido, esto se presenta por los retrasos en las estaciones, } \\
\text { dicho pedido se queda en el sistema. }\end{array}$ & $\begin{array}{l}\text { Al aplicar la política de mejora salieron } 16400 \text { cuellos y } 13505 \\
\text { puños de algodón debido a que las estaciones tuvieron una mayor } \\
\text { utilización y redujeron tiempos muertos. Esto representa un } \\
\text { incremento de producción del } 54,7 \% \text { y } 40.7 \% \text { respectivamente. }\end{array}$ \\
\hline
\end{tabular}

\section{REFERENCIAS}

[1] Valencia, M., Díaz, F., y Correa, J. C. Inventory planning with dynamic demand. A state of art review. DYNA, 183-191, 2015.

[2] Bernal, M. E., Cock, G., y Restrepo, J. H. Productividad en una celda de manufactura flexible simulada en Promodel utilizando "Path Networks type Crane". Tecnura, 133-144, 2015.

[3] Sánchez, C. Diseño de modelos de procesos productivos en ingeniería por simulación. Paideia XXI, 57-69, 2014.

[4] Gómez, R., Hernández, J. D., y Correa, A. Análisis de un sistema de producción de cárnicos utilizando simulación discreta. Espacios, 12-26, 2016.

[5] Sanchéz, P., Ceballos, F., y Sanchéz, G. Análisis del proceso productivo de una empresa de confecciones. Modelación y simulación. Ciencia e Ingeniería Neogranadina, 1-15, 2015.

[6] Herrera, J. C., Herrera, G., y Gonzalez, C. I. Mejora del proceso de fabricación de estibas de madera. Un caso de estudio. Ingeniería Solidaria, 1-21, 2017.
[7] Visbal Perez, E. T. Herramientas tecnológicas aplicables al Kanban para la optimización de los procesos en la empresa. Visión Gerencial, 82-104, 2016.

[8] Arango, M. D., Campuzano, L. F., y Zapata, J. A. Mejoramiento de procesos de manufactura utilizando Kanban. Revista Ingenierías Universidad de Medellín, 221-234, 2015.

[9] Parra, O. J. Sistemas de producción tipo Kanban: Descripción, componentes, diseño del sistema y bibliografía relacionada. Panorama, $11-22,2008$

[10] Curti, A. R., y Campos, R. R. De. Sistema Kanban Como Mecanismo De Controle De Processos. Revista Interface Tecnológica, 15(2), 208219. https://doi.org/10.31510/infa.v15i2.468. 2018.

[11] Murphy, A., Butterfield, J., y Higgins, P. Using Design Of Experiments To Define Factory Simulations For Manufacturing Investment Decisions. Queen's University Belfast, 1-9, 2017.

[12] Acheson, C., Mackle, D., Murphy, A., Butterfield, J., Higgins, P., Collins, R., y Tame, R. Using Design Of Experiments To Define Factory Simulations For Manufacturing Investment Decisions, 2017.

[13] Gómez, A., Quintana, N., y Ávila, J. O. Simulación de eventos discretos y líneas de balance, aplicadas al mejoramiento del proceso constructivo 
de la cimentación de un edificio. Ingeniería y Ciencia, 11(21),157-17 2015. https://doi.org/10.17230/ingciencia.11.21.8.

[14] Assadi, M., Zahraee, S., y Taghdisi, J. Integration of Computer Simulation, Design of Experiments and Particle Swarm Optimization to Optimize the Production Line Efficiency. International Journal of Swarm Intelligence and Evolutionary Computation, 135-139, 2016.

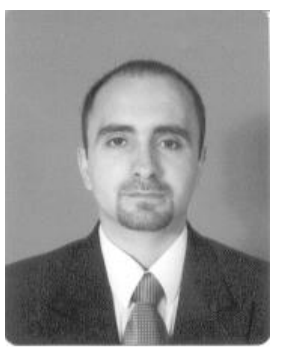

Sergio Augusto Fernández Henao es ingeniero Industrial de la Universidad Tecnológica de Pereira, Colombia, en junio de 2003. Magister en Investigación Operativa y Estadística de la Universidad Tecnológica de Pereira, Colombia, en julio de 2010. Actualmente se desempeña como docente vinculado e investigador en el programa de Ingeniería de Productividad y Calidad del Politécnico Colombiano Jaime Isaza Cadavid, Medellín, Antioquia. Integrante del Grupo de Investigación COINDE de la Facultad de Administración. Coordinador de la Red Académica de Productividad "REDPROD". Sus intereses en investigación son la optimización de procesos, la estadística aplicada, análisis envolvente de datos, simulación de eventos discretos y continuos, diseño de experimentos y lean manufacturing.

ORCID: https://orcid.org/0000-0002-3830-8102.

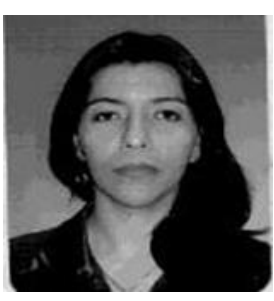

Angela Liceth Pérez Rendon es licenciada en Matemáticas y Física de la Universidad Tecnológica de Pereira, Colombia, en marzo de 2006. Magister en Investigación Operativa y Estadística de la Universidad Tecnológica de Pereira, Colombia, en mayo de 2016. Actualmente se desempeña como profesor tiempo completo e investigador en la Fundación Universitaria del área Andina. Pereira, Risaralda. Integrante activo del Grupo de Investigación (CIASA), del departamento de Ciencias Básicas. Sus intereses en investigación son la estadística aplicada, Investigación de Operaciones, Diseño Experimental. ORCID: https://orcid.org/0000-0002-2363-8782.

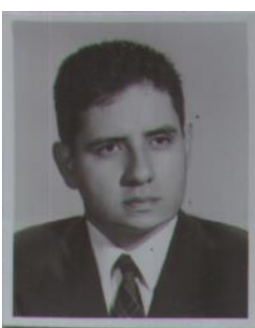

Pedro Daniel Medina Varela es ingeniero Mecánico de la Universidad del Valle, Colombia, abril de 1996. Magíster en Ingeniería Industrial de la Universidad de los Andes, Colombia. Actualmente profesor asociado a la Facultad de Ciencias Empresariales de la Universidad Tecnológica de Pereira (UTP) y director de la Maestría en Sistemas Integrados de Gestión de la Universidad Tecnológica de Pereira. Miembro del grupo de Investigación en Gestión de la Calidad y Normalización Técnica de la Facultad de Ciencias Empresariales de la UTP. Sus intereses de investigación enfocados en herramientas de mejoramiento organizacional, estadística aplicada y diseño de experimentos. 